\title{
Belphégor
}

\section{Fabio Negro, La Perla di Labuan. Una leggenda}

salgariana

\section{Vittorio Frigerio}

\section{(2) OpenEdition}

1 Journals

\section{Edizione digitale}

URL: http://journals.openedition.org/belphegor/547

DOI: 10.4000/belphegor.547

ISSN: $1499-7185$

Editore

LPCM

\section{Notizia bibliografica digitale}

Vittorio Frigerio, «Fabio Negro, La Perla di Labuan. Una leggenda salgariana », Belphégor [En ligne], 13-1 | 2015, mis en ligne le 09 mai 2015, consulté le 22 septembre 2020. URL : http://

journals.openedition.org/belphegor/547 ; DOI : https://doi.org/10.4000/belphegor.547

Questo documento è stato generato automaticamente il 22 settembre 2020.

\section{(c) (i) (9)}

Belphégor est mis à disposition selon les termes de la Licence Creative Commons Attribution - Pas d'Utilisation Commerciale - Pas de Modification 4.0 International. 


\section{Fabio Negro, La Perla di Labuan. Una leggenda salgariana}

\section{Vittorio Frigerio}

NOTIZIA

Negro, Fabio. La Perla di Labuan. Una leggenda salgariana. Piombino : Edizioni il Foglio, 2014. 195 p. ISBN : 978-88-7606-506-4 
1 Vi sono delle imprese al cui solo pensiero dovrebbe accapponarsi la pelle. Tra di esse possiamo contare la riconquista di Mompracem a bordo di una piccola flottiglia di prahos già provati da pericolosi scontri, e la scommessa di voler (ri)scrivere il sacro Graal dei romanzi salgariani: quella Perla di Labuan che l'Emilio stesso forse non scrisse mai, ma la cui leggenda, o il cui mito solca i mari dell'immaginazione dei lettori appassionati, sempre speranzosi di veder risorgere dai flutti del tempo - o dal fondo dimenticato di qualche cassetto - le avventure perdute della Tigre della Malesia e della sua sardonica e compassata spalla, il portoghese Yanez de Gomera.

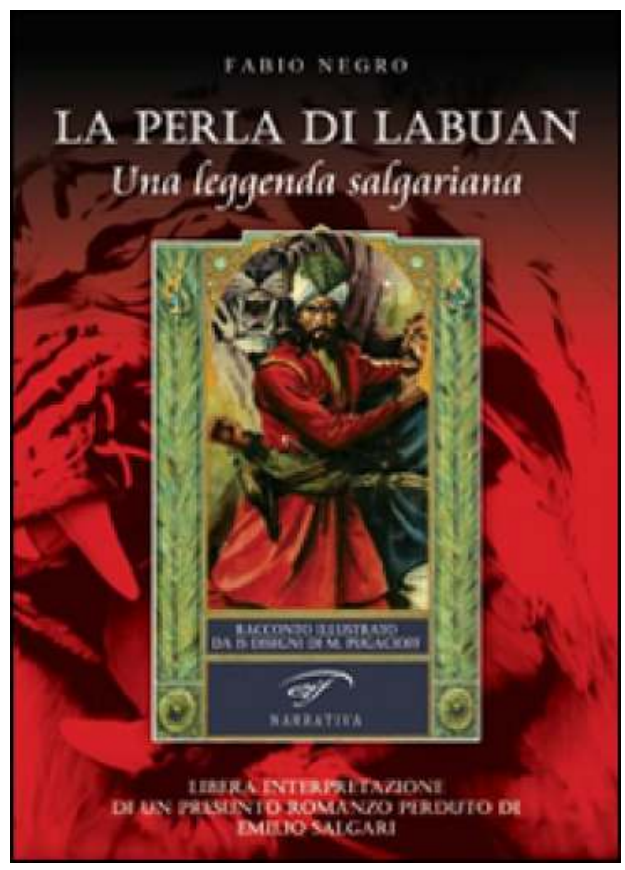

2 Ma riscrivere Salgari è un'avventura che coloro che sono cresciuti negli anni del dopoguerra - e finchè nei sessanta la televisione non è venuta adagio adagio a rimpiazzare nelle ore di svago la letttura appassionata dei racconti d'avventure - conoscono molto bene. Quanti romanzi trovati sugli scaffali delle biblioteche scolastiche o imprestati dopo molte insistenze a compagni riluttanti, portavano la firma di Emilio Salgari ma lasciavano nell'orecchio del lettore accorto la sensazione di una stonatura, l'impressione - giustissima peraltro! - di uno scarto sensibile tra quanto ci era ammannito e ciò che realmente sarebbe dovuto essere un romanzo colato dalla penna del maestro. E non bastava che tra quelle pagine riapparisse la sagoma tanto agognata del Corsaro Nero, o l'espressione cangiante tra ferocità e malinconia di Sandokan. Si intuiva, si capiva, si sapeva, che malgrado l'inganno editoriale quelle non erano pagine di Salgari. Ma si leggeva comunque, perchè il desiderio era sempre più forte della delusione e perchè qualche bella immagine, anche perduta tra paginate sciatte, poteva fungere da scintilla per riavviare il fuoco solo assopito dei ricordi.

3 Dopo tanti anni, Fabio Negro viene aggiungere il suo nome alla schiera di epigoni che si sono provati con alterne fortune a dare alle stampe opere di sapore salgariano. E lo fa mirando alto e proponendosi di completare idealmente un vuoto particolarmente difficile da colmare nella saga del personagggio forse più amato dell'epica salgariana. Questi non sono più tempi idonei per inganni editoriali, e il romanziere di oggi presenta la sua opera non solo dichiaratamente come un omaggio al grande antenato ("libera interpretazione di un presunto romanzo perduto di Emilio Salgari", si avvisa in copertina), ma corredata perfino da uno studio del più colto e curioso tra i ricercatori salgariani, Felice Pozzo, che rievoca con toni quasi da romanzo poliziesco la ricerca lunga e infruttuosa della "vera" Perla di Labuan, la cui esistenza effettiva finisce per negare malgrado tracce abbondanti quanto vaghe, e confuse o contraddittorie testimonianze. I suoi argomenti, va detto, sono purtroppo convincenti. Ma in un certo senso ciò non conta realmente più, perchè ora La Perla di Labuan esiste, e abbiamo Fabio Negro da ringraziare per questo. 
4 È superfluo dare un riassunto della trama, se non per dire che essa include la scena madre finora mancante nei romanzi dei pirati della Malesia: quella della morte di Marianna Guillonk, l'adorata compagna di Sandokan, rappresentata qui con un fedele miscuglio di dolcezza e di indomabile forza di carattere. Nel rispetto obbligato dei canoni salgariani il volume contiene anche le scene indispensabili di caccia, di battaglia, gli inseguimenti, le sorprese paradossalmente prevedibili ma sempre e lo stesso gratificanti, le liste parascientifiche di alberi e fiori dai nomi poeticamente impronunciabili, i dettagli topografici che ricreano un altrove d'altri tempi. Il tutto in un linguaggio convenientemente datato, con quel bel profumo di un italiano antiquato fedele a quello dell'originale financo in certi tic, nelle ripetizioni, in alcune costruzioni affrettate che pur nella loro rozzezza suonano però così meglio all'orecchio di quella lingua impoverita che regna oggidì nei media e spesso nel romanzo. Ma lo scrittore, giustamente, non ha potuto resistere del tutto alla legittima tentazione di fare proprio l'universo che andava ricreando. E così si scoprono segreti nuovi sul passato di Yanez, anch'essi verosimili e che prestano al Portoghese un altro, differente alone, oltre a quello che normalmente l'attornia, fatto del fumo delle sue sigarette. Alcuni tratti dei personaggi riescono più marcati forse che non talvolta nell'originale, tradendone la natura d'interpretazione moderna. Così l'indifferenza degli eroi alle religioni si fa esplicita, e l'anti-imperialismo del quale - a torto o a ragione - si è voluto fare una caratteristica principale salgariana risalta chiaramente, anche se nelle parole di un personaggio nuovo, l'olandese Wan Doren, che sostiene validamente i Nostri nei loro vagabondaggi marittimi. A questo riguardo val la pena di offrire la citazione integrale di uno scambio tra costui e l'amico di Sandokan :

"Ditemi, capitanoWan Doren, feceYanez, accendendosi una sigaretta. - Questa à una festa locale, di religione animista. Cosa vi ha a che fare il governo olandese?

- Oh ! - rispose l'altro. - È solo una forma di propaganda. Da una parte sfruttano e derubano il popolo, e da quell'altra se lo ingraziano partecipando alle loro solennità e versando cospicue somme ai loro sacerdoti.

- Siete incauto, - rise il Portoghese, - a parlare in questa maniera di coloro che guidano il vostro paese.

- Bah! Che volete che mi importi ? E del resto io mi riferisco non solo agli olandesi, ma agli inglesi, ai francesi, e agli spagnoli, che si stanno divorando questo mondo." (p. 118)

5 Amen, verrebe voglia d'aggiungere, tralasciando il fatto che giudizi espressamente politici sull'abbindolamento delle masse attraverso le arti della "propaganda" sembrano esulare un tantino dalla retorica originale salgariana. Ma l'interpretazione è più che legittima. Altri piccoli, discreti anacronismi offrono talvolta un sorriso, forse anche programmatico, così come la giustificazione per la diffusione del cholera (con la " $h$ " beninteso), che utilizza termini evocatori di dibattiti molto contemporanei : "Questo è il risultato di uno smodato sovraffollamento e di un flusso migratorio non controllato" (110, corsivo chiaramente nostro...).

Gli appassionati di Salgari leggeranno indubbiamente con gusto questo romanzo rispettoso delle sacre fonti, ma non dovuto a un pedissequo esecutore quanto a un coraggioso ed estroso creatore. Che si vuole di più per qualche ora di autentico piacere, dovutamente nostalgico ? Lasciamo perdere, in rivincita, le illustrazioni. Un Gamba o un Della Valle non si eguagliano facilmente.

7 Scampato all'ennesimo pericolo in compagnia del fedele Yanez, il Sandokan di Fabio Negro conclude sorridendo: "È già molte volte che la morte cerca di prenderci. Poi 
invece si spaventa e ci lascia tra i vivi" (143). Questo libro è un'altra prova che certi miti, decisamente, non muoiono mai.

\section{AUTORI}

\section{VITTORIO FRIGERIO}

Dalhousie University 